\title{
Scaling of wet granular flows in a rotating drum
}

\author{
Ahmed Jarray $^{1, *}$, Vanessa Magnanimo ${ }^{1}$, Marco Ramaioli ${ }^{2}$ and Stefan Luding ${ }^{1}$ \\ ${ }^{1}$ Multi Scale Mechanics, CTW, MESA+, University of Twente, Enschede, Netherlands \\ ${ }^{2}$ Department of Chemical and Process Engineering, University of Surrey, Guildford, United Kingdom
}

\begin{abstract}
In this work, we investigate the effect of capillary forces and particle size on wet granular flows and we propose a scaling methodology that ensures the conservation of the bed flow. We validate the scaling law experimentally by using different size glass beads with tunable capillary forces. The latter is obtained using mixtures of ethanol-water as interstitial liquid and by increasing the hydrophobicity of glass beads with an ad-hoc silanization procedure. The scaling methodology in the flow regimes considered (slipping, slumping and rolling) yields similar bed flow for different particle sizes including the angle of repose that normally increases when decreasing the particle size.
\end{abstract}

\section{Introduction}

A correct understanding of granular flow is critical to the success of a wide range of pharmaceutical processes including mixing, coating and tableting $[1,2]$.

Flow parameters are inherently scale-dependent: if the particle size is changed, characteristic properties such as the dynamic angle of repose (i.e. the angle near the midpoint of the flowing layer in a drum) and the depth of the free flowing layer will change. This makes the scaling of granular flows a complex endeavor.

A simple and practical geometry to study the flow of granular materials is the rotating drum. This has been extensively studied by many researchers including Taberlet et al. [3] who derived an equation to describe the $\mathrm{S}$ shape of the granular pile in a rotating drum. Rajchenbach [4] correlated the dynamic angle of repose to the rotation speed of the drum. Elperin and Vikhansky [5] proposed a model for describing the bed flow using a Mohr-Coulomb failure criterion.

While, most of these studies focused on the flow of dry particles, mechanisms governing particle flow in wet systems remain poorly understood. In fact, adding a small amount of liquid to a particulate system can significantly alter its flow behaviour. The work of Tegzes et al. [6] for instance showed that the capillary force determines the flow motion of particles. Brewster et al. [7] showed that the presence of interparticle cohesion reduces the concavity of the free flowing surface in the drum. Therefore, the effects of capillary force on wet granular flow cannot be ignored.

Dimensional analysis can be used to simplify the flow description by reducing the number of system parameters. Henein et al. [8] correlated the flow of particles in a drum to the magnitude of the Froude number and categorized it into six flow regimes; slipping, slumping, rolling, cascading, cataracting, and centrifuging. Orpe et al. [9] found that the flowing layer thickness is symmetric at low Froude number (Fr) and large particle size to drum radius $(r / R)$. However, so far, scaling relationships for wet particulate systems in a rotating drum are unavailable, and it is not clear how particle size affects the flow of particles in the presence of liquid-induced cohesion.
In this work, we study the flow of wet particles in a rotating drum in the slipping, slumping and rolling regimes by using a silanization method to alter the surface properties of the glass beads, and hence, to indirectly control the bed flow in the drum. We begin by investigating the influence of the particle size, rotation speed and the capillary force on the bed flow motion. Then, we propose an approach that ensures particle flow similarity for different particle sizes.

\section{Materials and Methods}

\subsection{Capillary force and silanization procedure}

The capillary force between interacting particles is known to control the flow behaviour of wet granular materials; here we focus on the pendular regime [10]. For a given particle radius $r$, the capillary force depends linearly on the contact angle $\theta$ and the surface tension $\gamma$.

$$
F^{c} \sim \gamma \cos \theta .
$$

For a proper understanding of the problem, we isolate the roles of particle size, contact angle, and surface tension. Similarly to the work of Raux et al. [11], we treat the surface of the glass beads via silanization to increase their hydrophobicity, that is the contact angle $\theta$. Using mixtures of ethanol-water as interstitial liquid and glass beads with different hydrophobicity, the capillary force between two adjacent beads can be manipulated over a wide range of values.

Chemical compounds used for silanization are: Silanization solution $\quad \mathrm{I} \sim 5 \% \quad(\mathrm{v} / \mathrm{v}) \quad(5 \% \quad(\mathrm{v} / \mathrm{v})$ dimethyldichlorosilane in Heptane, Selectophore), Hydrochloric acid (HCL, $0.1 \mathrm{M}$ ), Acetone and Ethanol.

The procedure for increasing the beads hydrophobicity is as follows: first, glass beads are cleaned for at least one hour by immersion into freshly prepared HCL solution under agitation using a rotorstator homogenizer. Then, they are rinsed thoroughly with deionized water and oven dried for 3 hours at $60^{\circ} \mathrm{C}$. Afterward, the freshly cleaned glass beads are immersed in $5 \%(\mathrm{v} / \mathrm{v})$ dimethyldichlorosilane in heptane (silanisation solution) under agitation for an hour. Finally, the glass beads are rinsed with acetone and allowed to air-dry under a fume hood for 24 hours. 


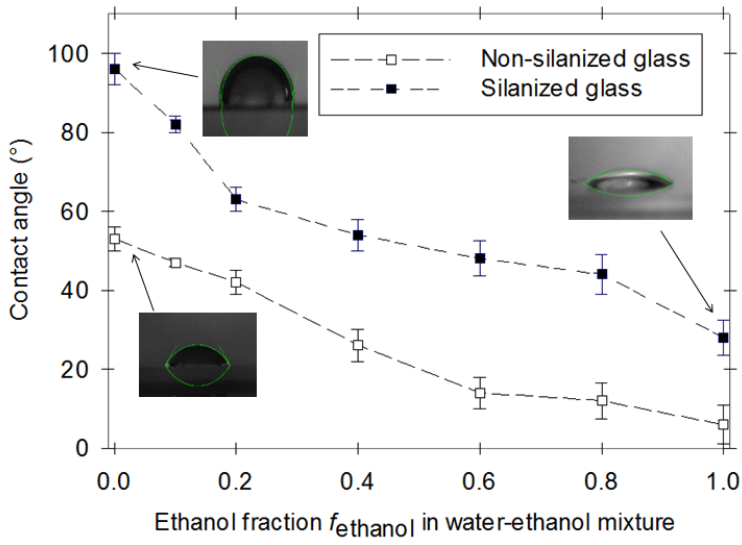

Fig. 1. Contact angles of water-ethanol mixtures as a function of the ethanol fraction on silanized and non-silanized glass.

To measure the contact angle $\theta$, liquid droplets are placed on a glass surface. Then, pictures of the droplet are taken using a MotionBLITZ EoSens camera with close-up lenses. The contact angle is then deduced by image analysis using LBADSA plugin in the open source imageJ software. The LBADSA plugin is based on the fitting of the Young-Laplace equation to the image data [12]. As shown in Fig. 1, water-ethanol mixtures with different ethanol fraction allow to obtain intermediate contact angles, which continuously decrease from $96^{\circ}$ to $30^{\circ}$ for silanized beads as the ethanol fraction in the liquid increases from 0 to 1 . The higher the contact angle, the lower the wettability of the glass.

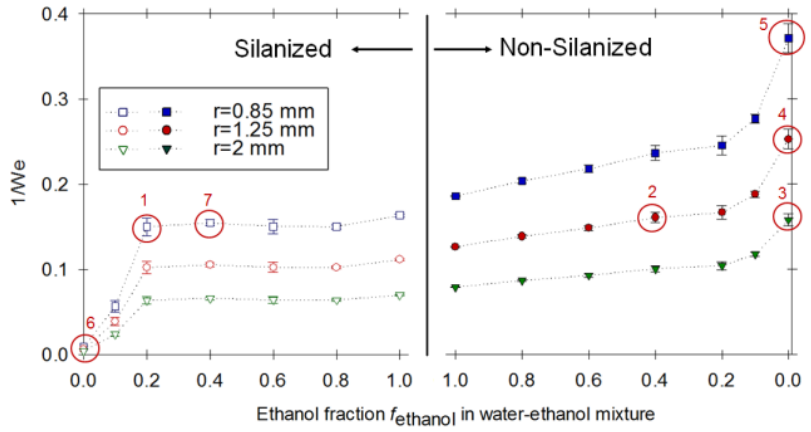

Fig. 2. Inverse of the Weber number $W e$ as a function of ethanol fraction for silanized and non-silanized glass for different particle radii $r$. The red circles mark to the configurations studied in section 3.3. Surface tension values are taken from Ref. [13].

To characterize the motion of wet particles, the particle based Weber number is often used [14, 15], given by the ratio of kinetic to capillary energy of the particle.

$$
W e=\frac{\rho_{p} r V^{2}}{\gamma \cos \theta}
$$

where $r$ the particle radius, $\rho_{p}$ the density of glass bead and $V$ the average velocity of the flowing particles. This velocity is taken constant, $V=0.11 \mathrm{~m} / \mathrm{s}$. The Weber number in equation (2) also takes into consideration the effect of the particle-liquid contact angle $\theta$.
In Fig. 2, we plot the inverse of the particle based Weber number We, defined in equation (2), against the volume fraction of ethanol in the water-ethanol mixture. Surface tension values are taken from Ref. [13]. Here, differently from e.g. $[14,15]$, the variability of the contact angle $\theta$ is taken into account. We vary $W e$ by varying the capillary force $F^{c} \sim \gamma \cos \theta$, for different particle sizes. The Weber number increases $\left(F^{c}\right.$ decreases) with increasing ethanol concentration for particles with unmodified glass surface, while We decreases ( $F^{c}$ increases) in the case of silanized glass beads, in agreement with the literature [16]. This allows to control the strength of the capillary force and $W e$ number in wet granular flows.

\subsection{The Drum apparatus}

Samples of silanized and non-silanized glass beads with different ethanol-water mixtures as described above, are then placed in a rotating drum to study the granular flow. The drum is made by a cylinder of 121 $\mathrm{mm}$ inner diameter, $22 \mathrm{~mm}$ width (frontal view in Fig. 3 a), and held between two circular plexiglass (PMMA) plates of $5 \mathrm{~mm}$ thickness to allow optical access. The drum is placed vertically on a rotating axle driven by a variable-speed motor. The PMMA walls of the drum are coated with FEB coating to prevent wet glass beads from sticking on the wall. Images of the rotating drum are recorded using a MotionBLITZ EoSens high speed camera working at a speed of 460 frame. $\mathrm{s}^{-1}$. Images are then post-processed using a particle tracking package in Matlab [17].

After each experiment, the drum is dried in an oven for one hour to let the remaining liquid from the previous experiment evaporate. In all experiments, the drum filling ratio is around $35 \%(\mathrm{w} / \mathrm{w})$ (i.e. $125 \mathrm{~g}$ of glass beads) and the amount of added liquid is $4 \mathrm{ml}$, where the pendular state is expected. a)

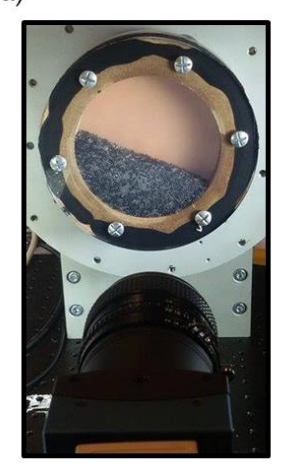

b)

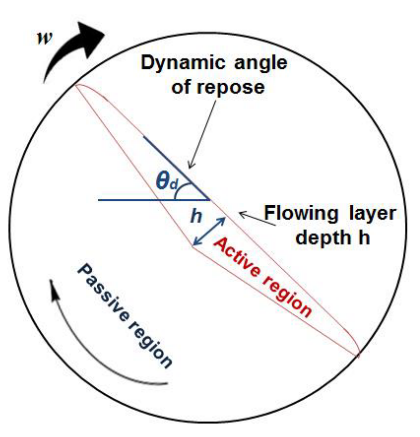

Fig. 3. a) Rotating drum apparatus (back) and camera (front), b) Schematic representation of the transverse plane the with the particle flow in a rotating drum.

We perform flow experiments in the drum for a selected set of particle types, with different hydrophobicity and sizes, namely $0.85 \mathrm{~mm}, 1.25 \mathrm{~mm}$ and $2 \mathrm{~mm}$.

Parameters and characteristics of the drum and the glass beads are summarized in Table 1 . 
Table 1. Properties of the drum and the glass beads.

\begin{tabular}{|c|c|}
\hline Properties & Value \\
\hline Drum, $\mathrm{R} \times \mathrm{L}(\mathrm{mm})$ & $60.5 \times 22$ \\
\hline Glass beads radii $r(\mathrm{~mm})$ & $0.85,1.25$ and 2 \\
\hline Rotation speed $(\mathrm{rpm})$ & 3 to 57 \\
\hline Particle density $\rho_{p},\left(\mathrm{~kg} \cdot \mathrm{m}^{-3}\right)$ & 2500 \\
\hline Filling level $\beta$ & $35 \%(125 \mathrm{~g})$ \\
\hline Volumetric liquid content $v_{l i q}(\mathrm{ml})$ & 4 \\
\hline
\end{tabular}

\section{Results and discussion}

\subsection{Effect of capillary force on the bed flow}

In order to characterize the flow in the drum, we focus on the dynamic angle of repose, i.e. the angle between the top surface of the rolling bed and the horizontal plane (Fig. 3 b). This angle is numerically computed by linear regression of the positions of the particles on the surface of the flow, detected by a concave-hull algorithm [18].

First, we investigate the flow in the simplest case of non-salinized particles with pure-water as interstitial fluid. This will help us to gain knowledge on the interplay between particle size and capillary force.

Fig. 4 shows the dynamic angle of repose versus rotation speed for three particle sizes in wet system with pure water as interstitial liquid (configuration 3, 4 and 5 in Fig. 2). As the rotation speed increases, the dynamic angle of repose increases. Smaller glass beads have a higher dynamic angle of repose. This is because a decrease of the particle size decreases the Weber number $W e$ (see Fig. 2), i.e. capillary forces become stronger.

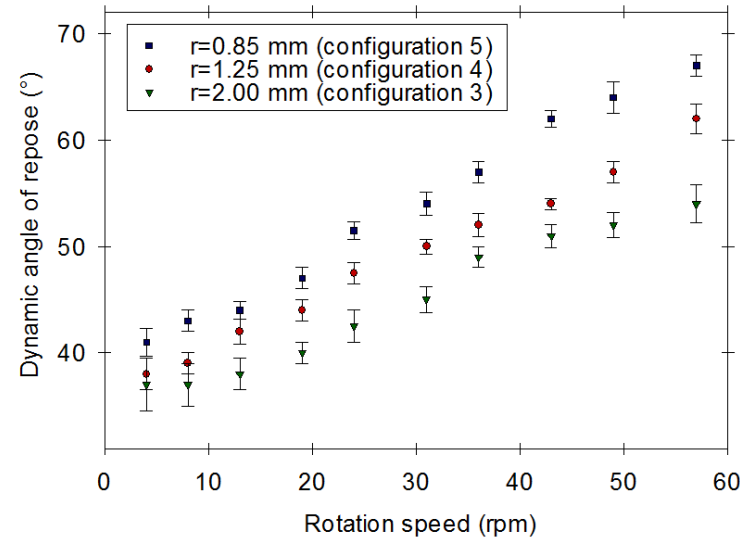

Fig. 4. Dynamic angle of repose as a function the rotation speed for different particle sizes in wet system.

In Fig. 5, we show the velocity gradient of the granular flow for particle size $1.25 \mathrm{~mm}$ at $25 \mathrm{rpm}$, with $0 \%$ ethanol concentration $\left(f_{\text {ethanol }}=0\right)$ in the case of wet glass beads (configuration 4 in Fig. 2) and silanized wet glass beads (configuration 6 in Fig. 2). For the sake of completeness, we also show the flow in the case of dry particles. The velocity profile of the particles in the drum is obtained using a matlab particle tracking method [17].

Clearly, the interparticle cohesion has a significant effect on the bed flow motion. Whereas the flow pattern has a convex shape for dry particles, an almost flat shape is observed for wet particles. The dynamic angle of repose for the wet particles shown in Fig. 5 (b) is about $46^{\circ}$, larger than that of dry particles (around $37^{\circ}$ in Fig. 5 a). By visual inspection of Fig. 5, we also notice that the depth of the high velocity zone becomes larger, but slower, in the wet case, suggesting a gradual transition from shear flow to plug flow. This transition is also marked by lower flow velocity, indicating a reduction of the mobility of particles.

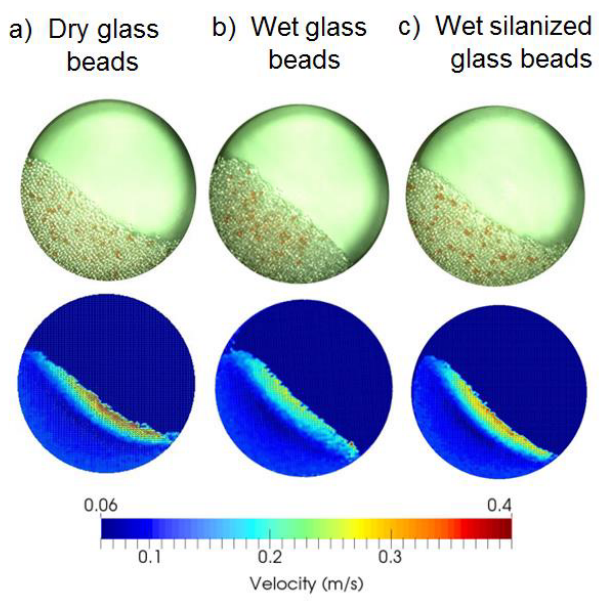

Fig. 5. Snapshots (top ) and velocity (bottom) of the bed in the rotating drum for a) dry, b) wet and c) silanized glass beads.

On the other hand, silanized glass beads mixed with water show a lower dynamic angle of repose and a thinner flowing layer (see Fig. $5 \mathrm{c}$ ), close to that of the dry case. This is because the capillary force becomes weaker in the case of silanized surface due to the higher contact angle. Thus, by means of silanization, it is possible to alter the bed flow via the contact angle and even approach the flow motion of a dry system.

\subsection{Scaling methodology}

The concept of similarity based on dimensional analysis states that two processes might be considered similar if all involved length scales are proportional, and all dimensionless numbers needed to describe them have the same value [19].

Five dimensionless groups are presented in equation (3) including Froude number $F_{r}$ (ratio of inertial force to gravity force), Weber number $W e$, liquid to particle volume ratio, capillary number $C_{a}$, and drum fill ratio $\beta$.

$$
\begin{aligned}
& F_{r}=\frac{2(R-r) \omega^{2}}{2 g}, W e=\frac{\rho_{p} r V^{2}}{\gamma \cos \theta}, \frac{N_{p} r^{3}}{v_{\text {liq }}} \\
& C_{a}=\frac{\mu V_{r}}{\gamma}, \beta
\end{aligned}
$$

where $N_{p}$ is the total number of particles, $\mu$ is the dynamic viscosity, and $V_{r}$ is the relative velocity between two particles in contact. In our study, we have kept the Froude number, liquid-particle volume ratio, and drum fill percentage constant among all experiments (see Table 1). In this study, we focus on the effect of the Weber number and neglect here the role of the Capillary number $\left(C_{a}<<1\right)$. 


\subsection{Particle size scaling}

Using the information from the previous sections, we propose a procedure to maintain dynamic similarity after scaling the particle size. In the case of granular flows, the relevant properties to compare are the dynamic angle of repose and the depth of the flowing layer. Here, we focus on the former and we test if we can obtain the same flow behavior by keeping the Weber number We constant. That is, for decreasing particle size, we must increase the capillary force accordingly.

We compare the flow motion of the smaller particle sizes $(1.25 \mathrm{~mm}$ and $0.85 \mathrm{~mm})$ to the flow of the $2 \mathrm{~mm}$ particles and we check whether they have the same dynamic angle of repose. For that, we refer to Fig. 2 and we keep the inverse of the Weber number $1 / W e \sim 0.16$ for the three cases, starting from configuration 3 with pure water. For particles with $r=0.85 \mathrm{~mm}$, rather than using water, we use a mixture of $20 \%$ ethanol- $80 \%$ water $(\mathrm{v} / \mathrm{v})$ with silanized $0.85 \mathrm{~mm}$ glass beads, indicated in Fig. 2 as configuration 2 . On the other hand, for particles of 1.25 $\mathrm{mm}$, we use $40 \%$ ethanol- $60 \%$ (v/v) water with nonsilanized beads, marked in Fig. 2 as configuration 1.

Fig. 6 presents the dynamic angle of repose of wet samples for different rotation speeds. While the open red symbols represent the configurations with almost the same Weber number, solid symbols represent configs. 4 and 5 with $100 \%(\mathrm{v} / \mathrm{v})$ water and lower Weber number.

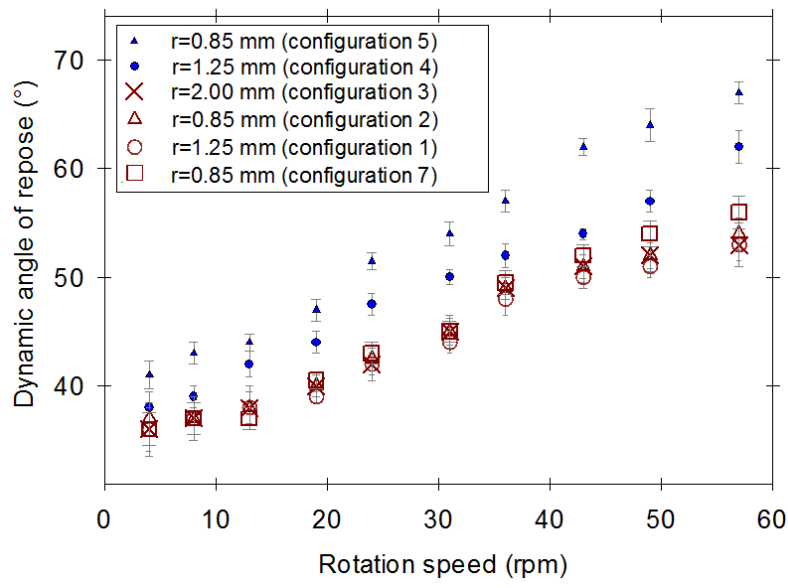

Fig. 6. Dynamic angle of repose as a function of the rotation speed for different particle sizes in wet systems.

The dynamic angle of repose of configurations 1 and 2 collapses with that of the $2 \mathrm{~mm}$ size case (config. 3 ), indicating that the latter can be rescaled to $0.85 \mathrm{~mm}$ or $1.25 \mathrm{~mm}$ sizes if the Weber number is kept constant, via the contact angle and the surface tension. This means that, experimentally, the scaling approach works in the considered flow regimes (slipping, slumping and rolling).

\section{Conclusion}

The effect of capillary force and particle size on the bed flow in a rotating drum was investigated at different rotation speeds, from 3 to $57 \mathrm{rpm}$. Specifically, we have shown that the presence of liquid increases the depth of the flowing layer and reduces the mobility of the particles. We established a procedure to alter the strength of capillary forces by using mixtures of ethanol-water as interstitial liquid with silanized and non-silanized glass beads. Then, we proposed a scaling methodology that ensures similarity of the dynamic angle of repose by keeping the Weber number constant after scaling the particle radius. We were able to obtain similar bed flow for different particle sizes, confirming that the proposed scaling methodology works for the few cases studied so far. A next step would be to test the scaling methodology on other quantities like the similarity of the flowing layer depth, and to include the effect of the liquid viscosity on the rather dynamic bed flow.

We thank H. Polman and B. Scheper for their help. Financial support through the "T-MAPPP" project of the European-Union-Funded Marie Curie Initial Training Network FP7 (ITN607453) is also acknowledged.

\section{References}

1. J. Osorio., F. Muzzio, Drug Dev. Ind. Pharm. 39, 1464-1475 (2013).

2. J. Prescott, R. Barnum, Pharm. Technol. 24, 60-85 (2000).

3. N. Taberlet, P. Richard, E.J. Hinch, Physical Review E 73, 050301 (2006).

4. J. Rajchenbach, Phys. Rev. Lett. 65, 2221 (1990).

5. T. Elperin and A. Vikhansky, Europhys. Lett. 42, 619 (1998).

6. P. Tegzes, T. Vicsek, P. Schiffer, Phys. Rev. E 67, 051303 (2003).

7. R. Brewster, G. S. Grest, and A. J. Levine, Physical Review E 79, 011305 (2009).

8. H. Henein, J. K. Brimacombe, and A.P. Watkinson Metall. Trans. A B 14, 191-205 (1983).

9. Orpe, A.V., Khakhar, D.V., Physical Review E 64, 031302 (2001).

10. Z. Fournier, D. Geromichalos, S. Herminghaus et al. J. Phys. Condens. Matter. 17, S477 (2005).

11. P. S. Raux, H. Cockenpot, M. Ramaioli, D. Quéré and C. Clanet, Langmuir 29, 11 (2013).

12. A.F. Stalder, T. Melchior, M. Müller, D. Sage, T. Blu, Colloids Surf., A 364, 72-81 (2010).

13. G. Vazquez, E. Alvarez, J. M. Navaza, J. Chem. Eng. Data , 40 (3), 611-614 (1995).

14. M. H. Klein Schaarsberg, I.R. Peters, M. Stern, K. Dodge et al., Phys. Rev. E 93, 062609 (2016).

15. Q. Xu, I. Peters, S. Wilken, E. Brown, and H. Jaeger. J. Vis. Exp. 85, 51249 (2014).

16. M. Tagawa, K. J. Gotoh, Adhes. Sci. Technol. 12, 1341 (1998).

17. Matlab Particle Tracking Code Repository, (2016), http://site.physics.georgetown.edu/matlab/index.htm

18. A. L. Chaua, X. Lia, W. Yu, Neurocomputing 122, 198-209 (2013).

19. F.M. Henderson, Open channel flow. Macmillan Publishing Co., Inc., (New York, 1966). 